\title{
Toxicity of methoprene as assessed by the use of a model microorganism
}

\author{
J.P. Monteiro ${ }^{\text {a }}$, A.S. Jurado ${ }^{\text {a,b,* }}$, A.J.M. Moreno ${ }^{\text {c }}$, V.M.C. Madeira ${ }^{\text {a }}$ \\ a Centro de Neurociências, Departamento de Zoologia, Universidade de Coimbra, 3004-517 Coimbra, Portugal \\ b Departamento de Bioquímica, Universidade de Coimbra, 3000 Coimbra, Portugal \\ ${ }^{\mathrm{c}}$ Instituto do Mar, Universidade de Coimbra, 3000 Coimbra, Portugal
}

Received 7 April 2005; accepted 17 June 2005

Available online 2 August 2005

\begin{abstract}
Methoprene is an insect juvenile growth hormone mimic, commonly used as a pesticide. Although widely used for the control of several pests, toxic effects on organisms of different phyla have been reported. These events triggered studies to clarify the mechanisms of toxicity of this insecticide putatively involved in ecological issues. Here we show the effect of methoprene on the normal cell growth and viability of a strain of the thermophilic eubacterium Bacillus stearothermophilus, previously used as a model for toxicological evaluation of other environment pollutants. Respiration studies were also carried out attempting to identify a putative target for the cytotoxic action of methoprene. Cell growth was affected and a decrease of the number of viable cells was observed as a result of the addition of methoprene to the growth medium, an effect reverted by the presence of $\mathrm{Ca}^{2+}$. Methoprene also inhibited the redox flow of $B$. stearothermophilus protoplasts before the cytochrome oxidase segment, an effect further studied by individually assessing the enzymatic activities of the respiratory complexes. This study suggests that methoprene membrane interaction and perturbation of cell bioenergetics may underlie the mechanism of toxicity of this compound in non-target organisms. (C) 2005 Elsevier Ltd. All rights reserved.
\end{abstract}

Keywords: Methoprene; Bacillus stearothermophilus; Bacterial growth; Lipid membrane; Respiratory activity

\section{Introduction}

Concerns about chemicals in the environment and their possible ecotoxic risks have emerged in the last decades. Methoprene (isopropyl $(2 E, 4 E)$-11-methoxy3,7,11-trimethyl-2,4-dodecadienoate), designed to mimic a juvenile growth hormone, was the first insect growth regulator approved by the Environmental Protection

Abbreviations: NADH, Nicotinamide adenine dinucleotide, reduced form; TMPD, $N, N, N^{\prime}, N^{\prime}$-Tetramethyl-p-phenylenediamine

* Corresponding author. Address: Centro de Neurociências, Departamento de Zoologia, Universidade de Coimbra, 3004-517 Coimbra, Portugal. Tel.: +351 239 834729; fax: +351 239826798 .

E-mail address: asjurado@ci.uc.pt (A.S. Jurado).
Agency (USA), in the 1970s, after extensive studies showing low toxicity to vertebrates (Zoecon Corporation, 1974) and rapid natural chemical degradation in the environment (Zoecon Corporation, 1973; Quistad et al., 1975) and through organism metabolism (Schooley et al., 1975; Quistad et al., 1975). However, deleterious effects induced by methoprene have been reported on non-target aquatic insects (Miura and Takahashi, 1973; Breaud et al., 1977), crustaceans (Breaud et al., 1977; Templeton and Laufer, 1983) and, at higher concentrations, some fish species (Quistad et al., 1976; McKague and Pridmore, 1978). These findings and an eventual relationship of methoprene with the increase of malformed amphibians in North America (LaClair et al., 1998; Stocum, 2000) motivated researches to 
reexamine the bioactivity of this compound and metabolites, in order to predict putative noxious effects on non-target organisms.

Alternative methods to animal testing for assessing chemical toxicity have been developed in the last decades. Mitochondria have provided data that have been correlated with cytotoxicity parameters derived from cell cultures and whole organisms (Blondin et al., 1987; Knobelock et al., 1990). Microorganisms, like yeasts (Haubenstricker et al., 1990) and bacteria (Sikkena et al., 1992; Carneiro de Melo et al., 1996), have also been used in toxicity tests that resulted in toxicity correlations concerning energy metabolism and cell viability (Sikkena et al., 1992; Donato et al., 1997b). A strain of the thermophilic eubacterium Bacillus stearothermophilus has been used as a model to study the cytotoxicity of several lipophilic drugs (Luxo et al., 2000; Rosa et al., 2000; Monteiro et al., 2003) and pollutants (Donato et al., 1997a,b; Martins et al., 2003) and a remarkable parallelism of effects on this prokaryotic model and other model systems (Donato et al., 1997a) has been observed. These results prompted us to use the strain of $B$. stearothermophilus to evaluate the toxicity of methoprene resulting from unspecific interactions with the cytoplasmic membrane. As a lipophilic molecule, methoprene may be incorporated into biomembranes, common components to all living systems and potential targets of xenobiotics. It is attempted to correlate the effects of methoprene on growth and cell viability of $B$. stearothermophilus with effects in vitro at the molecular level, viz. the interaction of the compound with the enzymatic complexes of the redox system.

\section{Materials and methods}

\subsection{Chemicals}

Methoprene $94.9 \%$ was obtained from Sigma-Aldrich Co. All the other chemicals were of the highest grade.

\subsection{Cultures}

The strain of Bacillus stearothermophilus and the conditions for its maintenance and growth have been described previously (Jurado et al., 1987). Liquid cultures were started with an early stationary inoculum and were grown in one liter Erlenmeyer flasks containing $200 \mathrm{ml}$ of diluted L-Broth supplemented or not with $2.5 \mathrm{mM} \mathrm{CaCl}_{2}$, at $65^{\circ} \mathrm{C}$ and shaken at $100 \mathrm{rpm}$ in a New Brunswick water bath shaker. Methoprene from a concentrated ethanolic solution was added to the growth medium to obtain concentrations ranging from 2.5 to $15 \mu \mathrm{M}$. Control cultures were grown in media without drug, but with $0.0375 \%$ (v/v) ethanol (i.e., the maximum amount of solvent used). The bacterial growth was measured by turbidimetry at $610 \mathrm{~nm}$ in a Baush \& Lomb Spectronic 21 spectrophotometer or by counting colony forming units (c.f.u.) on agar plates.

\subsection{Measurement of oxygen consumption rate}

Oxygen consumption was measured with protoplasts prepared from cells from cultures harvested in the middle of the exponential growth phase, by incubation with lysosyme, essentially as described by Wisdom and Welker (1973). The protein content of the protoplasts was determined by the Biuret method, calibrated with bovine serum albumin (Gornal et al., 1949). The respiratory activity of the protoplasts was monitored polarographically with a Clark oxygen electrode (Eastabrook, 1967) connected to a recorder, in a thermostated water-jacketed closed chamber $(1 \mathrm{~mL})$, with magnetic stirring, at $37^{\circ} \mathrm{C}$. Methoprene as an ethanolic solution was added to the reaction medium $(40 \mathrm{mM}$ Hepes-Tris, $10 \mathrm{mM}$ $\mathrm{MgCl}_{2}, \mathrm{pH}=7.5$ ) with the protoplasts ( $0.5 \mathrm{mg}$ protein), and each preparation was incubated for $4 \mathrm{~min}$ before the addition of the substrate. The effect of methoprene on oxygen consumption of protoplasts was examined using $10 \mathrm{mM}$ NADH or $10 \mathrm{mM}$ ascorbate plus $600 \mu \mathrm{M}$ TMPD as substrates.

\subsection{Effect of methoprene on the activities of redox complexes}

To assay the activities of the terminal segment (cytochrome oxidase), the segment including complexes I plus III (NADH-cytochrome $c$ reductase) of the redox chain of B. stearothermophilus and the $\mathrm{F}_{1}$ ATPase activity (reverse of $\mathrm{F}_{0} \mathrm{~F}_{1}$ activity), membranes were isolated from bacterial cells by sonication of cells with a sonicator Vibracell model VC-100 for $10-15$ min with $6 \mathrm{~s}$ pulses until the suspension became translucent. The membranes were collected by centrifugation at $148,000 \mathrm{~g}$ for $90 \mathrm{~min}$ at $20^{\circ} \mathrm{C}$ and the protein content was determined by the Biuret method (Gornal et al., 1949). The cytochrome oxidase activity was measured polarographically (Eastabrook, 1967); the respiratory activity of the segment including the complexes I plus III (NADH-cytochrome $c$ reductase) was measured spectrophotometrically by following the reduction of oxidized cytochrome $c$ as an increase in $A_{550}$. The $\mathrm{F}_{1}$ ATPase activity (reverse of ATP synthase activity) was determined by monitoring the change in $\mathrm{pH}$ associated with ATP hydrolysis with a system composed of a Crison $\mathrm{pH}$ meter and a potentiometric recorder calibrated by adding standard acid aliquots at the end of each experiment. All of the enzymatic activities were determined at $37^{\circ} \mathrm{C}$. The effect of methoprene on the enzymatic activities was studied by previous incubation of the preparations with the selected amounts of the 
compound for $4 \mathrm{~min}$; controls were prepared by adding volumes of ethanol corresponding to the maximum concentrations used in the enzymatic activity assays.

\subsection{Statistic analysis of data}

The results of specific growth rates and final cell densities are presented as means $\pm \mathrm{SD}$ of the number of experiments indicated. Multiple comparisons were performed using one-way ANOVA, with the Student-Newman-Keuls as a post-test. $P<0.05$ was considered significant.

\section{Results}

\subsection{Effects of methoprene on the growth of Bacillus stearothermophilus}

The strain of B. stearothermophilus used in this study was grown at $65^{\circ} \mathrm{C}$ in a complex medium (dilute LBroth) with an endogenous concentration of $0.115 \mu \mathrm{M}$ $\mathrm{Ca}^{2+}$ (basal-medium, Fig. 1A). The addition of $2.5 \mathrm{mM} \mathrm{CaCl} 2\left(\mathrm{Ca}^{2+}\right.$-supplemented medium $)$ at this temperature, i.e., the upper limit of the optimal temperature range (Jurado et al., 1987) stimulates growth, inducing a shorter lag period, an higher specific growth rate and a significantly higher final culture density (Table 1). This trend was counteracted by the addition of methoprene. Concentrations of $2.5-15 \mu \mathrm{M}$ methoprene inhibited growth in media with or without $\mathrm{Ca}^{2+}$ supplement, as documented in Fig. 1 and Table 1. Growth inhibition, characterised by progressively lower specific growth rates and lower bacterial yields, as a function of the insecticide concentration (Fig. 1 and Table 1), is attenuated by a $\mathrm{Ca}^{2+}$ supplement added to the culture medium (Table 1). Thus, the addition of $10 \mu \mathrm{M}$ methoprene to the basal-medium promoted a decrease of the specific growth rate to $57 \%$ and a decrease of the optical density in the stationary phase to $31 \%$. In comparison, the same concentration of methoprene added to a $\mathrm{Ca}^{2+}$-supplemented medium induced less severe effects (a reduction of the specific growth rate and the optical density to $90 \%$ and $83 \%$, respectively). Additionally, $15 \mu \mathrm{M}$ methoprene added to the basal-medium only allowed a very limited growth. In contrast, the specific growth rate and the amount of growth in the $\mathrm{Ca}^{2+}$-medium with $15 \mu \mathrm{M}$ methoprene were very similar or even higher than the correspondent growth parameters in cultures grown in the basal-medium without insecticide.

For a quantitative evaluation of methoprene inhibitory effects on cell viability, the number of viable cells was determined by counting the colony forming units (c.f.u.), after plating serial dilutions of the liquid cultures on agar plates. As shown in Fig. 1B, the number of viable cells after $30 \mathrm{~min}$ of incubation was practically the
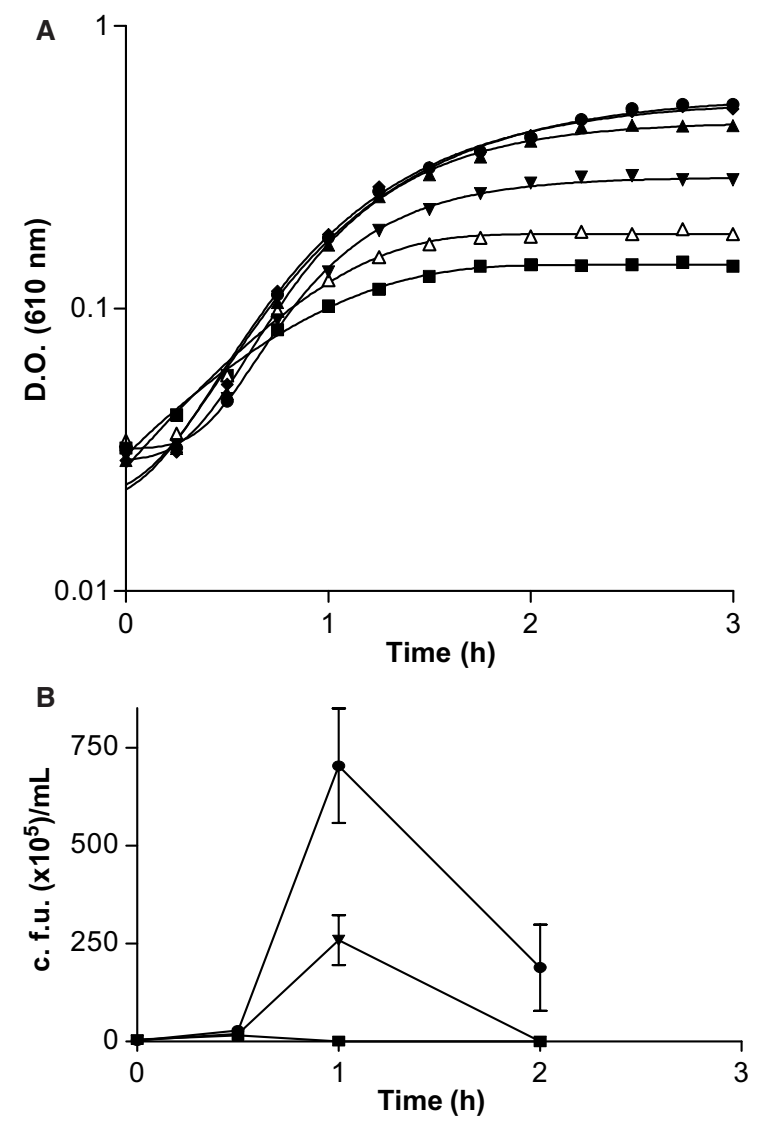

Fig. 1. (A) Effect of methoprene on the growth of cultures of Bacillus stearothermophilus, at $65^{\circ} \mathrm{C}$. Cells were grown in basal-medium (diluted L-Broth) without additives $(\bullet)$ and with $2.5 \mu \mathrm{M}(\checkmark), 5 \mu \mathrm{M}$ $(\mathbf{\Delta}), 7.5 \mu \mathrm{M}(\mathbf{\nabla}), 10 \mu \mathrm{M}(\triangle)$ and $15 \mu \mathrm{M}(\mathbf{\square})$ methoprene. (B) Effect of methoprene on the number of viable cells, capable of originating colonies when transferred to suitable solid growth medium; $(-)$ control

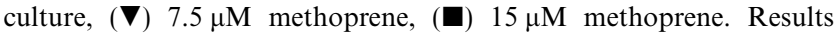
shown are typical of four (A) and three (B) independent experiments. Means \pm standard deviations of the number of colony forming units (c.f.u.) of triplicate determinations are represented.

same in cultures grown in the control medium and in media containing 7.5 and $15 \mu \mathrm{M}$ methoprene. However, within the next $30 \mathrm{~min}$, the culture in the control medium exhibited a comparatively higher increase of the number of viable cells, approaching a cell density approximately three times higher than the culture grown in a medium containing $7.5 \mu \mathrm{M}$ methoprene. At a concentration of $15 \mu \mathrm{M}$, methoprene exerted a deleterious effect on cells from the beginning of the incubation, completely vanishing cell viability after $1 \mathrm{~h}$. The corresponding turbidimetric curves (Fig. 1A) confirm that, by this time, cultures in the control medium and in a medium with $7.5 \mu \mathrm{M}$ methoprene are in the late exponential phase, whereas the culture with $15 \mu \mathrm{M}$ insecticide showed a limited increase of the optical density. After $2 \mathrm{~h}$ of incubation, cultures in the control medium and in a $7.5 \mu \mathrm{M}$ methoprene containing medium were in the stationary phase and the number of colony forming 
Table 1

Specific growth rates and cell densities (O.D.) reached in the stationary phase of cultures of Bacillus stearothermophilus grown in media supplemented $(+)$ or not $(-)$ with $2.5 \mathrm{mM} \mathrm{Ca}{ }^{2+}$, containing different concentrations of methoprene

\begin{tabular}{|c|c|c|c|}
\hline \multicolumn{2}{|c|}{ Additives to the growth medium } & \multirow[t]{2}{*}{ Specific growth rate $\left(h^{-1}\right)^{\mathrm{a}}$} & \multirow[t]{2}{*}{ Final cell density ( $\%$ of Control $)^{a}$} \\
\hline $\mathrm{Ca}^{2+}(2.5 \mathrm{mM})$ & Methoprene $(\mu \mathrm{M})$ & & \\
\hline- & 0.0 & $2.289 \pm 0.084(10)$ & $100 \pm 0(10)$ \\
\hline- & 2.5 & $2.246 \pm 0.022(5)$ n.s. & $90.674 \pm 4.778(5)^{*}$ \\
\hline- & 5.0 & $2.033 \pm 0.091(6)^{* * *, \circ \circ}$ & $69.981 \pm 5.275(6)^{* * *, \circ \circ \circ}$ \\
\hline- & 7.5 & $1.700 \pm 0.069(5)^{* * *, \infty \circ \circ}$ & $49.261 \pm 7.090(5)^{* * *, \circ \circ \circ}$ \\
\hline- & 10.0 & $1.312 \pm 0.111(9)^{* * *, \circ \circ \circ}$ & $30.781 \pm 6.375(10)^{* * *, \circ \circ \circ}$ \\
\hline- & 15.0 & $0.828 \pm 0.209(9)^{* * *, \circ \circ \circ}$ & $18.407 \pm 4.967(7)^{* * *, \circ \circ}$ \\
\hline+ & 0.0 & $2.508 \pm 0.036$ & $176.390 \pm 2.277(4)$ \\
\hline+ & 10.0 & $2.253 \pm 0.060(4)^{* *, \Delta \triangle \triangle}$ & $147.210 \pm 12.550(4)^{* * *, \triangle \triangle \triangle}$ \\
\hline+ & 15.0 & $2.187 \pm 0.094(4)^{* *, \Delta \Delta \Delta}$, n.s. & $128.330 \pm 17.515(4)^{* * *, \Delta \triangle \triangle, \circ \circ \circ}$ \\
\hline
\end{tabular}

${ }^{\text {a }}$ Values of specific growth rates and final cell densities are means \pm standard deviation from four or more independent experiments (number in brackets) and comparisons were performed using one way ANOVA, with the Student-Newman-Keuls as a post-test, for the following paired observations: control cultures (growth without additives) vs cultures grown in the basal-medium with different concentrations of methoprene or cultures grown in $\mathrm{Ca}^{2+}$-supplemented medium, without methoprene vs cultures with $\mathrm{Ca}^{2+}$ supplement plus 10 or $15 \mu \mathrm{M}$ methoprene (n.s., not significant; $\left.{ }^{* *} P<0.001 ;{ }^{* *} P<0.01,{ }^{*} P<0.05\right)$; cultures grown in the basal-medium with 10 or $15 \mu \mathrm{M}$ methoprene vs cultures with Ca ${ }^{2+}$ supplement plus 10 or $15 \mu \mathrm{M}$ methoprene, respectively $\left({ }^{\triangle \triangle} \mathrm{\triangle} P<0.001\right)$; cultures grown in the basal-medium with $5.0,7.5$, 10 and $15 \mu \mathrm{M}$ methoprene vs cultures grown in the basal-medium with $2.5,5.0,7.5$ and $10 \mu \mathrm{M}$ methoprene, respectively or cultures with Ca ${ }^{2+}$ supplement plus $15 \mu \mathrm{M}$ methoprene vs cultures with $\mathrm{Ca}^{2+}$ supplement plus $10 \mu \mathrm{M}$ methoprene $\left({ }^{\circ \circ} \mathrm{P}<0.001 ;{ }^{\circ \circ} \mathrm{P}<0.01\right.$; n.s., not significant $)$.

units significantly dropped. However, the control culture still contained a significant amount of viable cells, whereas in the methoprene inhibited culture, the number of cells was practically null.

\subsection{Effects of methoprene on the oxygen consumption rate of Bacillus stearothermophilus protoplasts}

The effects of methoprene on the oxygen consumption rate of protoplasts prepared from cells grown in the basal-medium are shown in Fig. 2. NADH and ascorbate-TMPD were used as respiratory substrates,

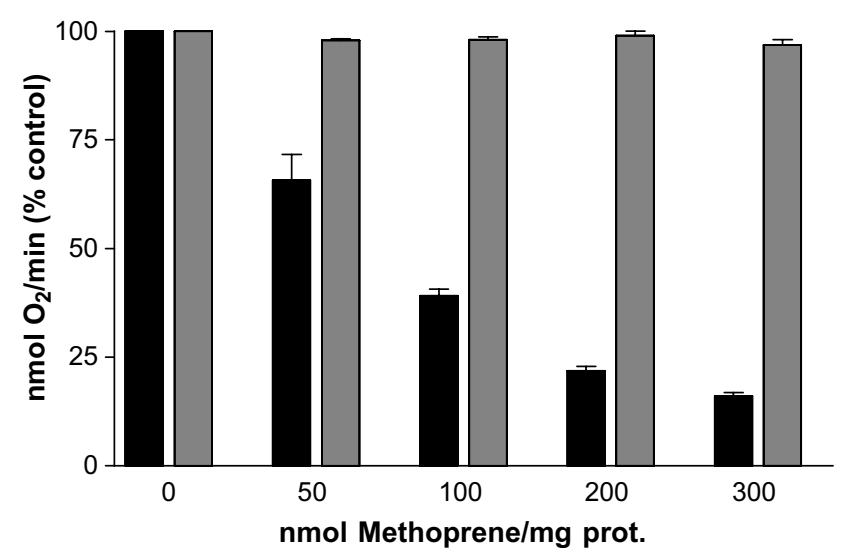

Fig. 2. Effect of methoprene on the oxygen consumption rate of Bacillus stearothermophilus protoplasts when $10 \mathrm{mM}$ NADH (black bars) or $10 \mathrm{mM}$ ascorbate- $600 \mu \mathrm{M}$ TMPD (grey bars) was used as the substrate. The bars show the levels of the respiratory rate as percentage of the control (protoplasts incubated for 4 min with an amount of ethanol equivalent to the larger volume of methoprene solution used). Data are means \pm standard deviations of three independent experiments. allowing to study the full respiratory system (NADH) or only the final oxidase segment (ascorbate-TMPD). When NADH was used as the substrate (Fig. 2), the addition of methoprene to the protoplasts significantly reduced the respiratory rate, as function of the concentration, in the range from 0 to $300 \mathrm{nmol} / \mathrm{mg}$ of protein. At the maximum concentration assayed, the respiratory rate was depressed to about $20 \%$ of the control. However, the oxygen consumption supported by ascorbateTMPD was insensitive to methoprene, in the same concentration range. As expected, the oxygen consumption was completely impaired by the addition of KCN $(1 \mathrm{mM})$, independently of the substrate used, reflecting the full inhibition of cytochrome oxidase.

\subsection{Effect of methoprene on the activities of redox complexes of the respiratory system of Bacillus stearothermophilus membranes}

To further clarify the effect of methoprene in the respiratory activity, membranes were isolated from bacterial cells and the activities of respiratory complexes were assessed independently. As expected from the $\mathrm{O}_{2}$ consumption data in protoplasts, Fig. 3 shows that increasing concentrations of methoprene (up to $300 \mathrm{nmol} / \mathrm{mg}$ of protein) induced a limited depression of the cytochrome oxidase activity, assessed in bacterial isolated membranes. On the other hand, methoprene addition to the membranes, up to a concentration of $200 \mathrm{nmol} / \mathrm{mg}$ of protein, promoted a considerable inhibition of the activity of the segment of the redox system including complexes I plus III (NADH-cytochrome $c$ reductase), evaluated by following the reduction of oxidized cytochrome $c$, using NADH as substrate. The $\mathrm{F}_{1}$ 


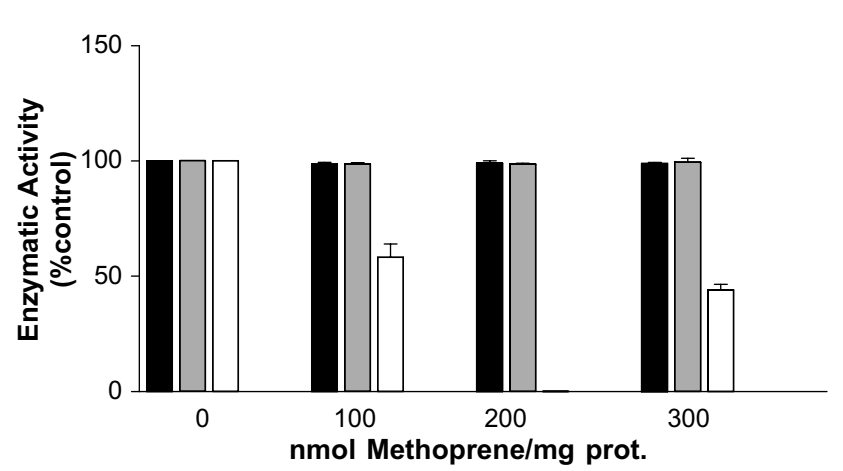

Fig. 3. Effects of methoprene on cytochrome oxidase (black bars), ATPase (grey bars) and the first segment of the respiratory chain (NADH dehydrogenase and cytochrome $c$ reductase-white bars) activities in isolated membranes of Bacillus stearothermophilus grown at $65^{\circ} \mathrm{C}$ in dilute L-Broth. The bars show the levels of the activities as percentage of the control values (without methoprene). Data are means \pm standard deviations of three independent experiments.

ATPase activity (reverse of ATP synthase) was not affected by methoprene (Fig. 3) in the studied concentration range $(0-300 \mathrm{nmol} / \mathrm{mg}$ protein $)$ and was completely eliminated by $12.5 \mu \mathrm{M} N, N^{\prime}$-dicyclohexylcarbodiimide confirming that the measured activity was related to the reversal of ATP synthase.

\section{Discussion}

Methoprene, as an insect growth regulator, was proposed as a safer replacement for the banned pesticide DDT in domestic and agricultural uses (Amos and Williams, 1977). However, several toxicological effects have been reported, varying across phyla (McKague and Pridmore, 1978; Templeton and Laufer, 1983). Methoprene is a non-cyclic synthetic terpenoid. Due to the lipophilic character, the interactions of this compound with membranes may issue non-specific toxic effects on non-target organisms. Microorganisms may provide suitable models to clarify cellular mechanisms of toxicity. B. stearothermophilus has been extensively used in our laboratory to study pesticide and drug toxicity mediated by membrane disturbance (Martins et al., 2003; Monteiro et al., 2003). In the present work, it was shown that methoprene perturbs growth of B. stearothermophilus. A lower viable count in the exponential and in the stationary phase was consistently observed in cultures containing the insecticide. The loss of cell viability in the stationary phase of methoprene inhibited culture was not evidenced by a decrease of turbidity, which suggests that methoprene, at the concentration used, does not induce cell lysis. On the other hand, the effects of methoprene on the bacterial growth showed to be dependent on the presence of divalent cations. The inhibition induced by low methoprene concentrations (2.5 and $5 \mu \mathrm{M})$ is completely abolished by the $\mathrm{Ca}^{2+}$ supple- ment to the growth medium (data not shown) and the highest concentrations of methoprene $(10$ and $15 \mu \mathrm{M})$ have limited inhibitory effect on growth of cultures in the $\mathrm{Ca}^{2+}$ supplemented medium, as compared to cultures in the basal-medium (Table 1). These observations concur well with previous studies in our laboratory with other lipophilic xenobiotics (Luxo et al., 2000; Donato et al., 1997a) suggesting that $\mathrm{Ca}^{2+}$, owing to its action as membrane stabiliser (Mosley et al., 1976; Jurado et al., 1991), may counteract the inhibitory effects on growth of xenobiotics that disturb membrane lipid organisation.

Attempting to correlate the impairment of cell viability and growth with perturbations of the membrane function, the respiratory activity was followed in protoplasts and the activities of respiratory complexes were assessed in membranes isolated from bacterial cells, as a function of methoprene concentration. Methoprene acts upstream the cytochrome oxidase segment, since the oxygen consumption of protoplasts was not inhibited when ascorbate-TMPD was used as respiratory substrate and only the activity of the initial segment (complexes I plus III) was affected by methoprene in membranes. ATPase activity (reverse of ATP synthase activity) was not affected by the presence of methoprene. Further clarification of the effects of methoprene in the respiratory system of B. stearothermophilus is technically difficult since there are not known inhibitors for the respiratory complexes, except for the final cytochrome oxidase $\left(\mathrm{CN}^{-}\right.$as inhibitor). Thus, additional studies on cell bioenergetics will be carried out to clarify at full extent the effect of the drug. Mitochondria emerge as a suitable model to complement experimental research, due to the extensive knowledge of the functioning of all the respiratory complexes. Preliminary studies carried out with rat liver mitochondria also showed methoprene interaction before cytochrome oxidase (unpublished data).

The data obtained with this bacterial model, besides directly helping to clarify the toxic action of methoprene, may also give hints on further studies to be carried out with other models for toxicological evaluation. Furthermore, the use of this bacterial model offers general advantages: (i) bacterial growth is easy and economic; (ii) ethical issues related with animal use and confirming its use as an alternative in vitro toxicity test; (iii) allows to correlate, in the same model, the toxicity of xenobiotic compounds as assessed in vivo by growth perturbations and in vitro effects on cell functions and physical perturbations of membranes.

\section{References}

Amos, T., Williams, P., 1977. Insect growth regulators: some effects of methoprene and hydroprene on productivity of several stored grain insects. Australian Journal of Zoology 25, 201-206. 
Blondin, G.A., Knobelock, L.M., Read, H.W., Harkin, J.M., 1987. Mammalian mitochondria as in vitro monitors of water quality. Bulletin of Environmental Contaminants Toxicology 38, 467-474.

Breaud, T.P., Farlow, J.E., Steelman, C.D., Schilling, P.E., 1977. Effects of the insect growth regulator methoprene on natural populations of aquatic organisms in Louisiana intermediate marsh habitats. Mosquito News 37, 704-712.

Carneiro de Melo, A.M.S., Cook, G.M., Miles, R.J., Poole, R.K., 1996. Nisin stimulates oxygen consumption by Staphylococcus aureus and Escherichia coli. Applied Environmental Microbiology $62,1831-1834$.

Donato, M.M., Jurado, A.S., Antunes-Madeira, M.C., Madeira, V.M.C., 1997a. Bacillus stearothermophilus as a model to evaluate membrane toxicity of a lipophilic environmental pollutant (DDT). Archives of Environmental Contamination and Toxicology 33, 109-116.

Donato, M.M., Jurado, A.S., Antunes-Madeira, M.C., Madeira, V.M.C., 1997b. Comparative study of the toxic actions of DDT and DDE on the growth and respiratory activity of a microorganism used as a model. Applied Environmental Microbiology 63, 4948-4951.

Eastabrook, R.W., 1967. Mitochondrial respiratory control and the polarographic measurement of ADP/O ratios. Methods in Enzymology 10, 41-47.

Gornal, A.G., Bardawill, C., David, M.M., 1949. Determination of serum proteins by means of the biuret method. Journal of Biological Chemistry 177, 751-756.

Haubenstricker, M.E., Meier, P.G., Mancy, K.H., Brabec, M.J., 1990. Rapid toxicity testing based on yeast respiratory activity. Bulletin of Environmental Contamination and Toxicology 44, 669-674.

Jurado, A.S., Santana, A.C., Costa, M.S., Madeira, V.M.C., 1987. Influence of divalent cations on the growth and morphology of Bacillus stearothermophilus. Journal of General Microbiology 133, 507-513.

Jurado, A.S., Pinheiro, T.J.T., Madeira, V.M.C., 1991. Physical studies on membrane-lipids of Bacillus stearothermophilus. Temperature and calcium effects. Archives of Biochemistry and Biophysics 289, 167-179.

Knobelock, L.M., Blondin, G.A., Read, H.W., Harkin, J.M., 1990. Assessment of chemical toxicity using mammalian mitochondrial electron transport particles. Archives of Environmental Contamination and Toxicology 19, 828-835.

LaClair, J.J., Bantle, J.A., Dumont, J., 1998. Photoproducts and metabolites of a common insect growth regulator produce developmental deformities in Xenopus. Environmental Science and Technology 32, 1453-1461.

Luxo, C., Jurado, A.S., Madeira, V.M.C., 2000. Toxicity assessment of tamoxifen by means of a bacterial model. Applied Biochemistry and Biotechnology 87, 219-232.

McKague, A.B., Pridmore, R.B., 1978. Toxicity of altosid and dimilin to juvenile rainbow trout and coho salmon. Bulletin of Environmental Contamination and Toxicology 20, 167-169.
Martins, J.D., Monteiro, J.P., Jurado, A.S., Madeira, V.M.C., 2003. Use of the microorganism Bacillus stearothermophilus as a model to evaluate toxicity of the lipophilic environmental pollutant endosulfan. Toxicology in vitro 17, 629-634.

Miura, T., Takahashi, R.M., 1973. Insect departmental inhibitors. 3. Effects on non-target aquatic organisms. Journal of Economic Entomology 66, 917-922.

Monteiro, J.P., Martins, J.D., Luxo, P.C., Jurado, A.S., Madeira, V.M.C., 2003. Molecular mechanisms of the metabolite 4-hydroxytamoxifen of the anticancer drug tamoxifen: use of a model microorganism. Toxicology in vitro 17, 629-634.

Mosley, G.A., Card, G.L., Koostra, W.L., 1976. Effect of calcium and anaerobiosis on the thermostability of Bacillus stearothermophilus. Canadian Journal of Microbiology 22, 468-474.

Quistad, R.B., Staiger, L.E., Schooley, D.A., 1975. Environmental degradation of the insect growth regulator methoprene (isopropyl (2E,4E)-11-methoxy-3,7,11-trimethyl-2,4-dodecanoate), III: photodecomposition. Journal of Agriculture and Food Chemistry 23, 299-303.

Quistad, G.B., Schooley, D.A., Staiger, L.E., Bergot, B.J., Sleight, B.H., Macek, K.J., 1976. Environmental degradation of the insect growth regulator methoprene. IX. Metabolism by bluegill fish. Pesticide Biochemical Physiology 6, 523-529.

Rosa, S.M.L.J., Antunes-Madeira, M.C., Jurado, A.S., Madeira, V.M.C., 2000. Amiodarone interactions with membrane lipids and growth of Bacillus stearothermophilus used as a model. Applied Biochemistry and Biotechnology 87, 165-175.

Schooley, D.A., Bergot, B.J., Dunham, L.L., Siddall, J.B., 1975. Environmental degradation of the insect growth regulator methoprene (isopropyl (2E,4E)-11-methoxy-3,7,11-trimethyl-2,4-dodecanoate), II: Metabolism by aquatic microorganisms. Journal of Agriculture and Food Chemistry 23, 293-298.

Sikkena, J., Poolman, B., Konings, W.N., de Bont, J.A.M., 1992. Effects of the membrane action of tetralin on the functional na structural properties of artificial and bacterial membranes. Journal of Bacteriology 174, 2986-2992.

Stocum, D.L., 2000. Frog limb deformities: an "Eco-Devo" riddle wrapped in multiple hypotheses surrounded by insufficient data. Teratology 62, 147-150.

Templeton, N.S., Laufer, H., 1983. The effects of a juvenile hormone analog (Altosid ZR-515) on the reproduction and development of Daphnia magna (Crustacea: Cladocera). International Journal of Invertebrate Reproduction 6, 99-110.

Wisdom, C., Welker, N.E., 1973. Membranes of Bacillus stearothermophilus: factors affecting protoplasts stability and thermostability of alkaline phosphatase and reduced nicotinamide adenine dinucleotide oxidase. Journal of Bacteriology 114, 13361345.

Zoecon Corporation, 1973. Technical bulletin on Altosid: Environmental Properties.

Zoecon Corporation, 1974. Technical bulletin on Altosid: Toxicological Properties. 